\section{Commentary: Relax and breathe}

\section{Todd L. Demmy, MD}

The multicenter randomized clinical trial reported by Liu and colleagues ${ }^{1}$ challenges the operative comfort zones of thoracic surgeons accustomed to selective positive pressure ventilation. For thoracic surgeons and anesthesiologists to relax enough to let their patients breathe spontaneously during minimally invasive surgery, sufficient equipoise and potential benefits are needed to stoke the desires for disruptive technologies.

First, we should suppress our natural tendency dismiss this trial's results because its study population was different from our own. Notably, body mass index was relatively low and patient health (including pulmonary function) was relatively high. Yet, this was the case preference by thoracic surgeons during the early experiences of modern thoracoscopic surgery. Another issue was that mechanical pleurodesis did not accompany these operations. Yet, there are data suggesting that, although popular, scarification may not be necessary. ${ }^{2}$

So what theoretical advantages exist to warrant disrupting reasonably safe surgical anesthesia systems that provide quiet operative fields for challenging procedures? Fundamentally, natural physiologic mechanisms are innately biocompatible and likely to clear secretions, balance ventilation/perfusion, and signal warning if not overly suppressed. Mechanical ventilation of any duration is associated with nosocomial infection, especially with the uncertainty of bronchial toilet during selective intubation. Selective intubation requires deeper anesthesia that reduces the need for local anesthetic or regional blocks required by sedated patients. However, these blocks also reduce the downregulation of spinal inhibitory neurons which can cause acute and prolonged pain. ${ }^{3}$ Prolonged videoassisted thoracoscopic surgery operations may have more

\footnotetext{
From the Department of Thoracic Surgery, Roswell Park Cancer Institute, Buffalo, NY; and the Department of Surgery, University at Buffalo, Buffalo, NY. Disclosures: The author reported no conflicts of interest.

The Journal policy requires editors and reviewers to disclose conflicts of interest and to decline handling or reviewing manuscripts for which they may have a conflict of interest. The editors and reviewers of this article have no conflicts of interest.

Received for publication Feb 1, 2021; revisions received Feb 1, 2021; accepted for publication Feb 2, 2021; available ahead of print Feb 12, 2021.

Address for reprints: Todd L. Demmy, MD, Department of Thoracic Surgery, Roswell Park Cancer Institute, Elm and Carlton Sts, Buffalo, NY 14263 (E-mail: todd. demmy@ roswellpark.org).

J Thorac Cardiovasc Surg 2022;163:1715-6

$0022-5223 / \$ 36.00$

Copyright (c) 2021 by The American Association for Thoracic Surgery https://doi.org/10.1016/j.jtcvs.2021.02.009
}

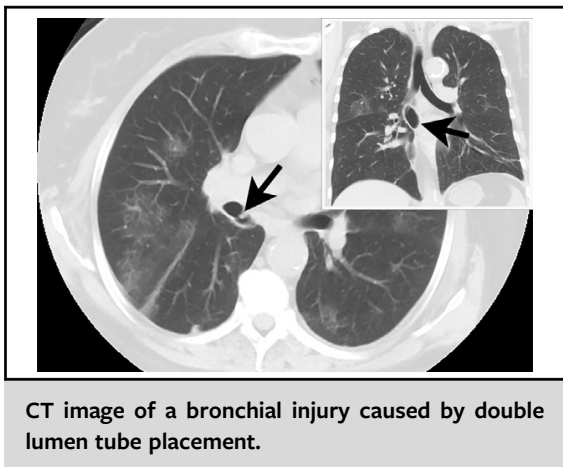

CENTRAL MESSAGE

Spontaneous, nonintubated patient ventilation is another step toward less-invasive thoracic surgery and is comparable to previous challenges that have been overcome.

associated complications because of synergistic mechanisms like amplified pain and other pneumonia-promoting etiologies. ${ }^{4}$

What if? failure scenarios are major psychologic barriers for experienced professionals. Fortunately, low-risk airway patients can be intubated safely in lateral decubitus positions using standard, laryngeal mask, or various endoscopic techniques. ${ }^{5,6}$ An advanced bronchial blocker or exchange catheter for double-lumen intubation then follows. Temporary wound closure with adhesive barrier dressing allows rapid repositioning, if necessary. Severe complications of airway conversions are possible, but are they really more intimidating than thoracotomies for video-assisted thoracoscopic surgery bleeding? On balance, are such bad scenarios going to be more common than double-lumen tube complications caused by immediate perforations (Figure 1) or glottic dysfunctions leading to aspiration in older adults. ${ }^{7,8}$

Programmatically, this effort requires anesthesiologists who are willing to extend their efforts toward the above interests. Such work promotes dedicated thoracic anesthesiology teams, which are also desirable. Also, these services parallel or expand medical thoracoscopic programs often driven by interventional pulmonologists. Once established, frail patients could benefit even more by extending these less-invasive programs. Successful, nonintubated thoracic surgery programs have been established for a long time in the United States at innovative centers like the Geisinger Health System. ${ }^{9}$ 


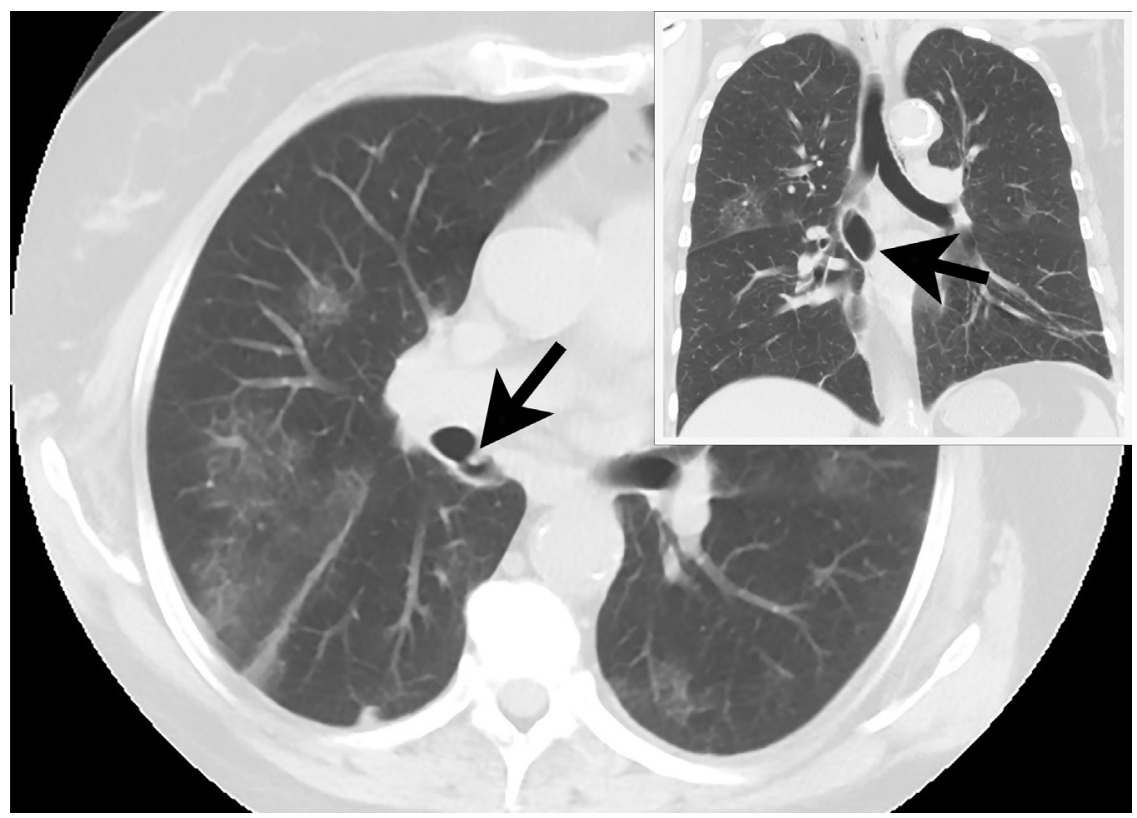

FIGURE 1. Computed tomography image of a bronchus intermedius injury caused by double lumen intubation that, while contained, required cancellation of the planned left-sided video-assisted thoracoscopic surgery procedure (arrow indicates site of perforation on axial image and contained pneumomediastinum on coronal view).

Now that our anesthesia and surgeon colleagues have produced level-one evidence for us to ponder, it is reasonable to consider adopting the skill of operating on anesthetized, spontaneously breathing patients. We should see if avoiding selective ventilation could benefit specific individuals or our practice as a whole.

\section{References}

1. Liu J, Liang H, Cui F, Liu H, Zhu C, Liang W, et al. Spontaneous versus mechanical ventilation during video-assisted thoracoscopic surgery for spontaneous pneumothorax: a randomized trial. J Thorac Cardiovasc Surg. 2022;163:1702-14.e7. 2. Vuong NL, Elshafay A, Thao LP, Abdalla AR, Mohyeldin IA, Elsabaa K, et al. Efficacy of treatments in primary spontaneous pneumothorax: a systematic review and network meta-analysis of randomized clinical trials. Respir Med. 2018;137:152-66.

3. Nosotti M, Rosso L, Tosi D, Palleschi A, Mendogni P, Righi I, et al. Preventive analgesia in thoracic surgery: controlled, randomized, double-blinded study. Eur J Cardiothorac Surg. 2015;48:428-33.
4. Dexter E, Attwood K, Demmy T, Yendamuri S. Does increased operative duration of lobectomy for early stage lung cancer increase perioperative morbidity? Presented at the Society of Thoracic Surgeons 57th Annual (Virtual) Meeting, January 29-31, 2021.

5. Ambrogi MC, Fanucchi O, Korasidis S, Davini F, Gemignani R, Guarracino F, et al. Nonintubated thoracoscopic pulmonary nodule resection under spontaneous breathing anesthesia with laryngeal mask. Innovations (Phila). 2014;9:276-80.

6. Gao Y, Lin B, Huang J, Lin X, Lin C. Flexible video endoscope versus Macintosh laryngoscope for orotracheal tracheal intubation in the lateral position: a study protocol for a randomized controlled trial. Trials. 2019;20:166.

7. Knoll H, Ziegeler S, Schreiber JU, Buchinger H, Bialas P, Semyonov K, et al. Airway injuries after one-lung ventilation: a comparison between double-lumen tube and endobronchial blocker: a randomized, prospective, controlled trial. Anesthesiology. 2006;105:471-7.

8. Tsai MH, Ku SC, Wang TG, Hsiao TY, Lee JJ, Chan DC, et al. Swallowing dysfunction following endotracheal intubation: age matters. Medicine (Baltimore). 2016;95:e3871.

9. Katlic MR, Facktor MA. Video-assisted thoracic surgery utilizing local anesthesia and sedation: 384 consecutive cases. Ann Thorac Surg. 2010;90:240-5. 